\title{
Spin frequency distributions of binary millisecond pulsars
}

\author{
A. Papitto ${ }^{1}$, D. F. Torres ${ }^{1,2}$, N. Rea ${ }^{1,3}$, and T. M. Tauris ${ }^{4,5}$ \\ 1 Institut de Ciències de l'Espai (IEEC-CSIC), Campus UAB, Fac. de Ciències, Torre C5, parell, 2a planta, 08193 Barcelona, Spain \\ e-mail: papitto@ice.csic.es \\ 2 Institució Catalana de Recerca i Estudis Avançats (ICREA), 08010 Barcelona, Spain \\ 3 Astronomical Institute "Anton Pannekoek", University of Amsterdam, Postbus 94249, 1090-GE Amsterdam, The Netherlands \\ 4 Argelander-Institut für Astronomie, Universität Bonn, Auf dem Hügel 71, 53121 Bonn, Germany \\ 5 Max-Planck-Institut für Radioastronomie, Auf dem Hügel 69, 53121 Bonn, Germany
}

Received 17 April 2013 / Accepted 27 April 2014

\begin{abstract}
Rotation-powered millisecond radio pulsars have been spun up to their present spin period by a $10^{8}-10^{9} \mathrm{yr}$ long X-ray-bright phase of accretion of matter and angular momentum in a low-to-intermediate mass binary system. Recently, the discovery of transitional pulsars that alternate cyclically between accretion and rotation-powered states on time scales of a few years or shorter, has demonstrated this evolutionary scenario. Here, we present a thorough statistical analysis of the spin distributions of the various classes of millisecond pulsars to assess the evolution of their spin period between the different stages. Accreting sources that showed oscillations exclusively during thermonuclear type I X-ray bursts (nuclear-powered millisecond pulsars) are found to be significantly faster than rotationpowered sources, while accreting sources that possess a magnetosphere and show coherent pulsations (accreting millisecond pulsars) are not. On the other hand, if accreting millisecond pulsars and eclipsing rotation-powered millisecond pulsars form a common class of transitional pulsars, these are shown to have a spin distribution intermediate between the faster nuclear-powered millisecond pulsars and the slower non-eclipsing rotation-powered millisecond pulsars. We interpret these findings in terms of a spin-down due to the decreasing mass-accretion rate during the latest stages of the accretion phase, and in terms of the different orbital evolutionary channels mapped by the various classes of pulsars. We summarize possible instrumental selection effects, showing that even if an unbiased sample of pulsars is still lacking, their influence on the results of the presented analysis is reduced by recent improvements in instrumentation and searching techniques.
\end{abstract}

Key words. accretion, accretion disks - magnetic fields - pulsars: general - stars: neutron - stars: rotation - X-rays: binaries

\section{Introduction}

The accretion of $0.1-0.2 M_{\odot}$ transferred by a companion star through an accretion disc in a low-mass X-ray binary (LMXB) is the widely accepted process to explain old neutron stars (NSs) that spin at a period of a few milliseconds (Alpar et al. 1982; Radhakrishnan \& Srinivasan 1982; Bhattacharya \& van den Heuvel 1991). This framework is known as the recycling scenario and identifies accreting X-ray bright NSs as the progenitors of radio-millisecond pulsars whose emission is powered by their loss of rotational energy. In this scenario, after a 0.1-10 Gyr long X-ray-bright accretion phase that spins up the NS (see, e.g., Tauris et al. 2012), the pressure exerted by the NS magnetic field is able to sweep out the light-cylinder volume, sparking the rotation-powered pulsed radio emission of the recycled pulsar. Mass-accretion not only spun up the $\gtrsim 300$ binary and isolated rotation-powered millisecond pulsars known in the Galaxy, but probably also reduced their magnetic field strength to values of $10^{7}-10^{9} \mathrm{G}$ (see, e.g., Bhattacharya \& Srinivasan 1995 , for a review).

The accretion-driven spin up of an NS in an LMXB is demonstrated by the observation of coherent pulsations at a period of a few ms from 15 NSs (accreting millisecond pulsars, hereafter accreting MSPs; Wijnands \& van der Klis 1998; Patruno \& Watts 2012), and of quasi-coherent-oscillations observed from an additional ten sources exclusively during thermonuclear type I X-ray bursts (nuclear-powered millisecond pulsars, hereafter nuclear $\operatorname{MSPs}^{1}$; Chakrabarty et al. 2003; Watts 2012). The swings between rotation and accretionpowered behaviour recently observed from IGR J18245-2452 (Papitto et al. 2013; Pallanca et al. 2013; Ferrigno et al. 2014; Linares et al. 2014) PSR J1023+0038 (Archibald et al. 2009; Stappers et al. 2013; Patruno et al. 2014), and XSS J12270-4859 (Bassa et al. 2014; Papitto et al. 2014; Roy et al. 2014; Bogdanov et al. 2014) demonstrated that NSs in some LMXBs alternate cyclically between rotation and accretion-powered states on short time-scales of a few years, or shorter.

In this paper, we compare the spin frequency distributions of the various samples of MSPs discovered so far, to test theories for describing the spin evolution of NSs as they evolve from the accretion to the rotation-powered stages of the recycling scenario (see, e.g., Tauris 2012, and references therein).

\section{Population of rotation- and accretion-powered binary millisecond pulsars}

To rely on the most recent sample of the rapidly growing population of rotation-powered millisecond pulsars (hereafter RMSPs), we considered the lists of radio MSPs compiled by

1 We stress that here, the term nuclear only refers to the mechanism that powers the emission of the source while it shows pulsations, whereas for most of the time, the X-ray output is due to mass accretion. 
Table 1. Samples of binary millisecond pulsars.

\begin{tabular}{lrcccl}
\hline \hline Class & $N$ & $\langle v\rangle(\mathrm{Hz})$ & $\tilde{v}(\mathrm{~Hz})$ & $\sigma_{v}(\mathrm{~Hz})$ & Description \\
\hline RMSPs & 199 & 268 & 274 & 139 & Rotation-powered (radio) pulsars in a binary system \\
Galactic RMSPs & 128 & 262 & 267 & 141 & RMSPs in the Galactic field \\
cluster RMSPs & 71 & 277 & 276 & 138 & RMSPs of globular clusters \\
eclipsing RMSPs & 42 & 367 & 377 & 154 & RMSPs whose radio-pulsed emission is irregularly eclipsed \\
non-eclipsing RMSPs & 157 & 242 & 252 & 122 & RMSPs whose radio-pulsed emission is not eclipsed \\
LMXBs & 25 & 413 & 401 & 150 & All accretion-powered pulsars (accreting MSPs + nuclear MSPs) \\
accreting MSPs & 15 & 353 & 377 & 140 & Accreting millisecond pulsars (X-ray coherent oscillations) \\
nuclear MSPs & 10 & 502 & 552 & 123 & Nuclear-powered millisecond pulsars (only X-ray burst oscillations) \\
transitional MSPs & 56 & 365 & 377 & 150 & Transitional pulsars (accreting MSPs + eclipsing RMSPs) \\
\hline
\end{tabular}

Notes. Number of objects, mean, median and standard deviation, of the spin frequency distributions of the considered classes of rotation and accretion-powered millisecond pulsars $(P<30 \mathrm{~ms})$ in binary systems. IGR J18245-2452 is counted as both an eclipsing- and an accreting MSP.

D. R. Lorimer ${ }^{2}$ and P. C. C. Freire ${ }^{3}$, corresponding to sources found in the Galactic field and globular clusters, respectively. As of January 2014, these lists contain 339 radio pulsars with a spin period shorter than $30 \mathrm{~ms}$. Slightly fewer than half of them are isolated sources, or systems for which an orbital period could not yet be determined. In the following, we only consider binary RMSPs to compare them with their accreting progenitors, since the evolutionary history of isolated MSPs might have been somewhat different than that of pulsars with a companion star (e.g. Verbunt et al. 1987; Kluzniak et al. 1988). The binary RMSPs that show irregular eclipses of their radio emission (Roberts 2013, hereafter eclipsing RMSPs) share a close link with their accreting progenitors. In fact, the observed eclipses are due to absorption caused by matter lost by the companion star that is ejected from the system by irradiation of the pulsar (Fruchter et al. 1988; Kluzniak et al. 1988). Because the semi-degenerate companion star of at least some of these eclipsing pulsars is very close to filling their Roche lobe (see, e.g., Breton et al. 2013), the mass lost by these stars may then occasionally overcome the pressure of the radio pulsar, driving the system into an accretion state. Switches between accretion- and rotation-powered stages have recently been observed from three eclipsing RMSPs (Archibald et al. 2009; Papitto et al. 2013; Patruno et al. 2014; Bassa et al. 2014). We summarize the properties of the various populations of binary RMSPs in Table 1 and plot the distribution of their frequencies in the top panel of Fig. 1.

The spin period is known for 25 accreting NSs in LMXBs (15 accreting MSPs, 10 nuclear MSPs, cf. Sect. 1). All accreting MSPs and seven out of ten nuclear MSPs are X-ray transients, accreting at a rate up to $10^{-9} M_{\odot} \mathrm{yr}^{-1}$ only during their fewweeks-long outbursts, and are found in quiescence at levels corresponding to less than $<10^{-12} M_{\odot} \mathrm{yr}^{-1}$ for most of the time (e.g. Coriat et al. 2012, and references therein). Even though these two classes overlap (burst oscillations were also observed from eight accreting MSPs), we considered sources that showed oscillations only during bursts (nuclear MSPs) to form a distinct class, because so far they did not show evidence of possessing a magnetosphere able to channel the accreted mass close to the magnetic poles and yield coherent pulsations. Nuclear MSP bursts tend to be observed in brighter states that are characterized by a softer spectrum, while they occur even in the hard state in accreting MSPs (Galloway et al. 2008; Watts et al. 2009; Watts 2012), which possibly indicates that an important role is played

\footnotetext{
2 Available from http://astro.phys.wvu.edu/GalacticMSPs

3 Available from http://www.naic.edu/ pfreire/GCpsr.html
}

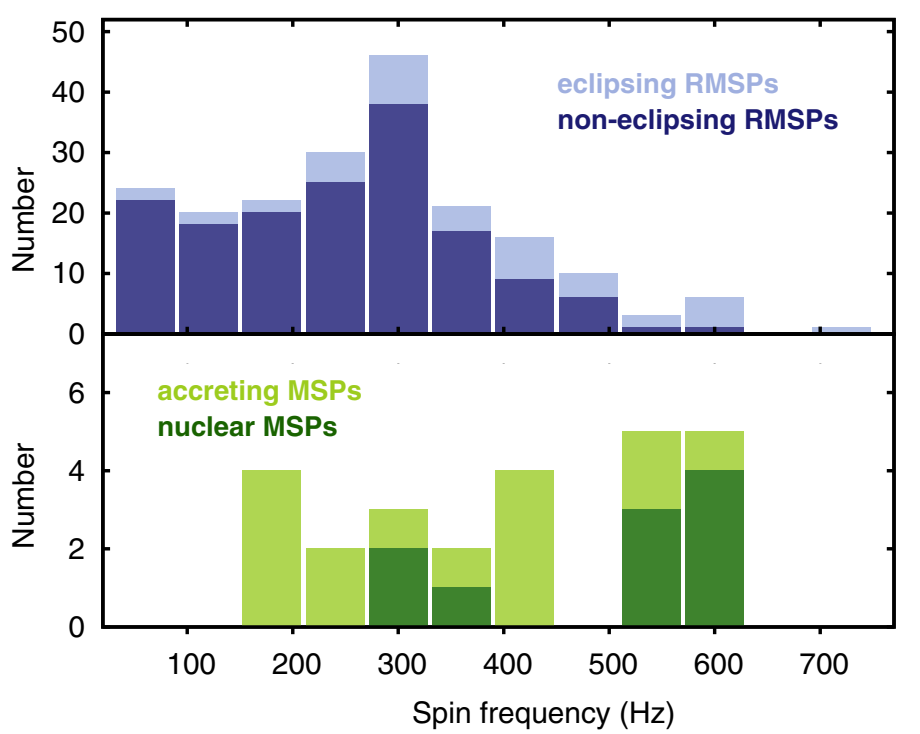

Fig. 1. Spin frequency distribution of binary non-eclipsing and eclipsing rotation-powered millisecond pulsars (top panel; blue and light blue), and nuclear-powered and accreting millisecond pulsars (bottom panel; green and light-green).

by the magnetosphere in determining burst oscillation properties. The properties of the spin distribution of LMXB pulsars are summarized in Table 1 and are plotted in the bottom panel of Fig. 1.

The recent discovery of systems that switch between rotation- and accretion-powered states on short time-scales also motivated us to define an intermediate class of systems, dubbed transitional pulsars (transitional MSPs). These systems behave as eclipsing RMSPs when the mass in-flow rate is low and the pressure of the pulsar prevents mass from falling onto the NS, while they are seen as X-ray-bright accreting sources when the transferred mass overcomes this pressure. IGR J182452452, observed as an accreting MSP and an eclipsing RMSP at different times (Papitto et al. 2013), is the prototype of this class. A transition to a rotation-powered state during X-ray quiescence has also been proposed for a number of accreting MSPs on the basis of the observed spin-down (Hartman et al. 2008, 2009, 2011; Patruno 2010; Papitto et al. 2011; Riggio et al. 2011; Patruno et al. 2012b), reprocessed optical emission (Burderi et al. 2003, 2009; Campana et al. 2004; Di Salvo et al. 2008; D' Avanzo et al. 2009), and orbital evolution 


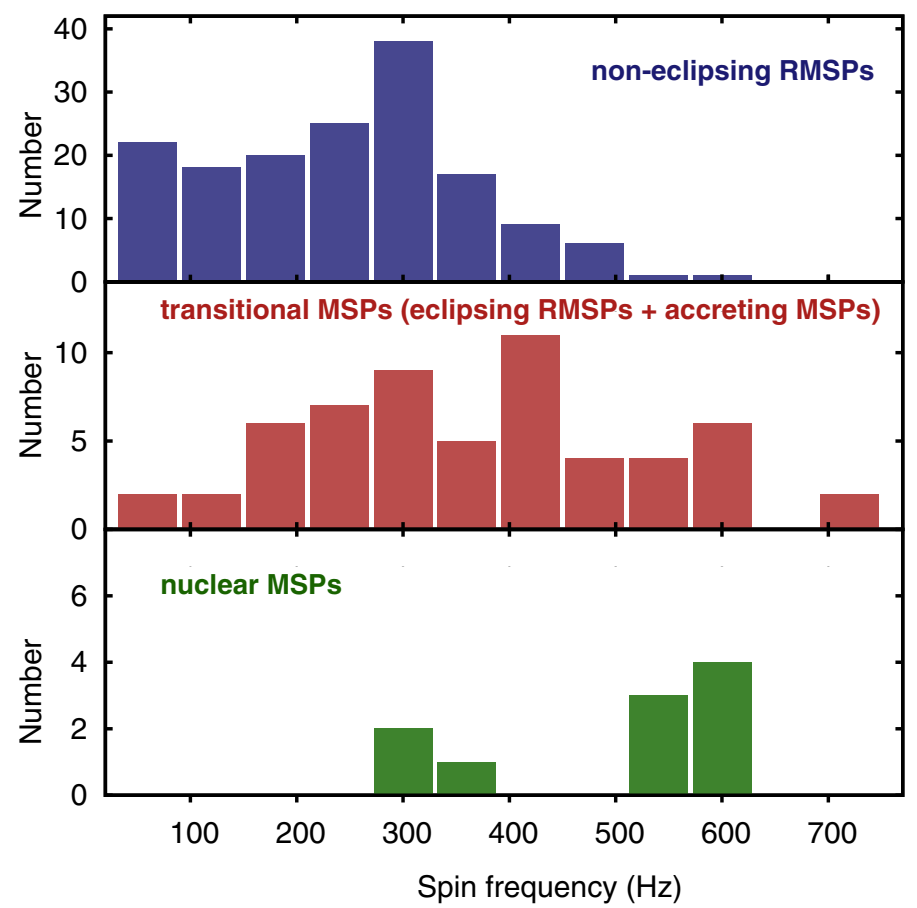

Fig. 2. Spin frequency distribution of non-eclipsing RMSPs (blue), transitional MSPs (defined as the sum of eclipsing RMSPs and accreting MSPs; red) and nuclear MSPs (green).

(Di Salvo et al. 2008; Burderi et al. 2009; Patruno et al. 2012b), even if radio and $\gamma$-ray pulsations have not been detected so far (Burgay et al. 2003; Iacolina et al. 2009, 2010; Xing \& Wang 2013). On the other hand, state transitions to an accretion stage have only been observed from eclipsing RMSPs (see above); these sources are the only possible candidates to undergo such fast transitions because the companion star has to spill matter through the inner Lagrangian point of the orbit to do so. Hence, we define the class of transitional MSPs as the sum of accreting MSPs and eclipsing RMSPs. The spin distribution of this class is plotted in the middle panel of Fig. 2, where the distribution of non-eclipsing millisecond radio pulsars (non-eclipsing RMSPs) and nuclear MSPs are also shown.

We note that only sources with a spin period shorter than $30 \mathrm{~ms}$ are considered in these samples because we aim at comparing only pulsars that are at (or close to) the end of the recycling process. While the exact value of this threshold is somewhat arbitrary, a significantly shorter period may indicate that the spin-up process has been interrupted halfway or has recently been activated, which may occur in the dense environment of globular clusters where dynamical exchange interactions are relatively frequent (Verbunt et al. 1987). This criterion excludes of an accreting source such as the $11 \mathrm{~Hz}$ pulsar IGR 17480-2446 in Terzan 5, which is thought to be just mildly recycled, having initiated its spin-up phase shorter than about $10 \mathrm{Myr}$ ago (see, e.g. Patruno et al. 2012a).

\section{Comparison of the spin distributions}

To compare the spin frequency distributions of the various classes defined in the previous section, we relied on the Kuiper test (Kuiper 1960; Stephens 1974; Paltani 2004). A Student t-test (as applied by Tauris 2012) is inadeguate to compare the various spin distributions because most of these spin frequency distributions are very different than a normal distribution, as for example can be shown with a Shapiro-Wilk test. The Kuiper test is preferred over the Kolmogorov-Smirnov (used, e.g., by Hessels 2008), because it is equally sensitive to deviations close to the median and towards the tails of the distribution.

The spin distributions of binary rotation-powered millisecond pulsars in the Galactic field (Galactic RMSPs) and those belonging to a globular cluster (clusters RMSPs) are similar. The probability that the two ensembles are drawn from the same distribution is 0.28 , and we therefore considered them as a single sample. On the other hand, eclipsing RMSPs and non-eclipsing RMSPs have a significantly different spin distribution. The former group has a mean spin frequency higher by $\sim 125 \mathrm{~Hz}$, and the probability that the two ensembles share the same parent distribution is $8.5 \times 10^{-4}$.

A glance at Table 1 and Figs. 1 and 2 might lead one to conclude that accreting MSPs are faster than binary millisecond radio pulsars (RMSPs), as noted by several authors (Ferrario \& Wickramasinghe 2007; Hessels 2008; Tauris 2012). However, even though accreting MSPs are on-average faster than RMSPs by $\sim 100 \mathrm{~Hz}$, comparing the two distributions gives a probability of 0.374 that they come from the same parent distribution, which clearly does not allow us to identify any significant difference. To determine the influence of the paucity of known accreting MSPs on this result, we simulated $10^{7}$ samples of $\mathcal{N}$ objects with spin frequencies based on a normal distribution with the same mean and standard deviation as known accreting MSPs (see Table 1). We then varied the number of simulated objects $(\mathcal{N})$ and re-ran the simulations. To detect a $3 \sigma$ difference to the RMSP distribution in 90 per cent of the $10^{7}$ simulations we ran, there need to be at least $\mathcal{N}=50$ accreting MSPs. We conclude that if the spin frequency distributions of accreting MSPs and RMSPs are different, we would be able to detect this difference only when the number of known accreting MSPs has increased by more than a factor of three (assuming a normal distribution).

On the other hand, accreting sources that showed pulsations only during type-I X-ray bursts (nuclear MSPs) are the fastest class of objects among those defined in this work. Their average frequency is roughly twice as fast as that of RMSPs and $\sim 90 \mathrm{~Hz}$ higher than that of accreting MSPs. The difference to RMSPs is significant because the probability that they come from the same distribution is $2.5 \times 10^{-3}$. The probability that the samples of accreting MSPs and nuclear MSPs come from the same parent distribution is 0.154 , although this value is hardly constraining; we have performed equivalent simulations as explained above and found that each sample needs at least 60 objects for a Kuiper test to identify a statistically significant spin difference between these two classes.

The total LMXB sample of accreting MSPs and nuclear MSPs has a higher average frequency than RMSPs, with a probability of only $\lesssim 1$ per cent that the two samples share the same distribution.

Accreting-MSPs and eclipsing RMSPs have a very similar spin distribution (see Table 1). The difference between their average frequencies is just $14 \mathrm{~Hz}$, and the probability that the two samples come from the same parent distribution is 0.912 . This is compatible with the hypothesis that they form a common class of objects at the same evolutionary epoch, which we labelled transitional MSPs. From comparing transitional MSPs with noneclipsing RMSPs we find that the probability that the two distributions are compatible is $3.9 \times 10^{-5}$, which indicates a significant difference. A low probability (0.048) is also found when transitional MSPs are compared with the faster nuclear MSPs. The tentatively defined class of transitional MSPs therefore has 


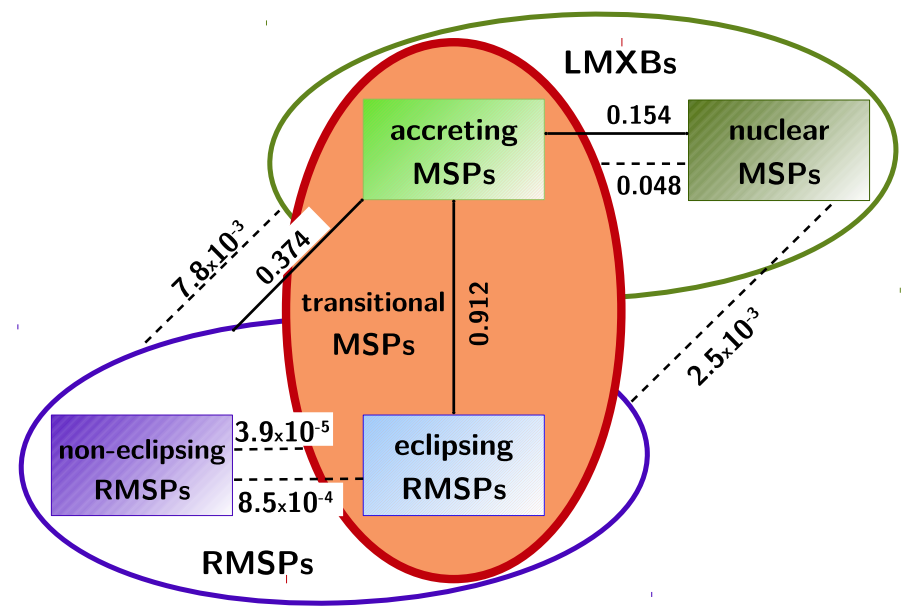

Frequency $(\mathrm{Hz})$

Fig. 3. Relational diagram of the various samples defined in this work, based on the comparison of their spin frequency distributions. Dashed lines connect samples that have a probability lower than 5 per cent of coming from the same parent distribution, while solid lines connect those with a higher probability. Numbers give the probability that the considered samples are drawn from the same distributions, evaluated with a Kuiper test.

a spin frequency distribution intermediate between that of nuclear MSPs and non-eclipsing RMSPs.

We summarize our results in the relational diagram plotted in Fig. 3, where dashed lines connect samples with a probability lower than $5 \%$ of being drawn from the same parent distribution, while solid lines connect those for which we evaluated a higher probability.

\section{Discussion}

Our analysis shows that nuclear MSPs form the only class of accretion-powered pulsars that are significantly faster than RMSPs, while accreting MSPs are not. However, if accreting MSPs and eclipsing RMSPs are assumed to form a common class of transitional pulsars, this class is characterized by an spin frequency distribution intermediate between faster nuclear MSPs and slower non-eclipsing radio pulsars.

The frequency difference between nuclear MSPs and RMSPs $(\gtrsim 230 \mathrm{~Hz}$ ) cannot be due to the weak magneto-dipole spin-down torque that acts onto the NS during the rotation-powered pulsar phase because this develops on a long time-scale, $\tau_{\mathrm{em}} \approx 10 \mathrm{Gyr}$ (for an NS spinning at $500 \mathrm{~Hz}$, with a magnetic dipole of $10^{26} \mathrm{G} \mathrm{cm}^{3}$ ). The spin-down torque that developes during the latest stages of the accretion phase, caused by the interaction between the NS magnetic field and the plasma rotating in the disc, is much stronger (up to a factor of 100; Ghosh \& Lamb 1979). As this torque is more intense when the NS is faster $\left(\propto v^{2}\right.$, e.g., Rappaport et al. 2004), it eventually balances the rate at which the accreted matter delivers its specific angular momentum to the NS $\left(\propto v^{-1 / 3}\right)$. This allows us to define an equilibrium period at which the overall torque acting onto the NS vanishes, the exact value of which depends on assumptions on the disc structure and how the field is twisted by the differential rotation of the disc plasma (Davidson \& Ostriker 1973; Ghosh \& Lamb 1979; Wang 1987; Bozzo et al. 2009; see, e.g., Ghosh 2007 for a review). Here, we considered the estimate given by Kluźniak \& Rappaport (2007) for a $1.4 M_{\odot}$ NS accreting from an optically thick, gas-pressure-dominated disc, as

$v_{\text {eq }}^{\text {(magn) }} \simeq 723 \mu_{26}^{-6 / 7} \dot{M}_{-10}^{3 / 7} \mathrm{~Hz}$,

where $\mu_{26}$ is the NS magnetic dipole in units of $10^{26} \mathrm{G} \mathrm{cm}^{3}$, and $\dot{M}_{-10}$ is the mass accretion rate in units of $10^{-10} M_{\odot} \mathrm{yr}^{-1}$. We note that this estimate does not take into account the possible ejection of matter by the rotating magnetosphere (see below). At the end of the accretion stage, the companion star decouples from its Roche lobe over a typical time-scale of $\approx 100 \mathrm{Myr}$ (Tauris 2012). If the NS would spin at the magnetic equilibrium until the complete turn-off of mass accretion, this would result in a spin-down of the NS well below the observed spin frequencies (Ruderman et al. 1989). A departure from spin equilibrium must therefore occur at some point. Tauris (2012) showed that the spin equilibrium is broken by the rapidly expanding magnetosphere resulting from the significant decrease in the ram pressure of the transferred material when the donor star terminates its mass-transfer phase. The resulting spin-down is aided by the onset of a propeller phase (Illarionov \& Sunyaev 1975) that acts to bring the NS farther out of equilibrium during the Roche-lobe decoupling phase.

Depending mainly on the magnetic field strength of the accreting pulsar, the loss of rotational energy computed by Tauris (2012) typically amounted to about 50 per cent, corresponding to a decrease in spin frequency of a factor $\sqrt{2}$, when the recycled pulsars switch from the accretor to the final long-term rotation-powered state. This value is of the order of the difference observed between the spin distribution of nuclear MSPs and RMSPs, making the spin-down experienced by the NS during the permanent decoupling of the companion star from its Roche lobe a viable explanation.

The spin distribution of accreting MSPs, on the other hand, is closer to that of RMSPs than to nuclear MSPs. This might originate from the differences between the two classes of accretionpowered sources. Unlike nuclear MSPs, accreting MSPs possess a magnetosphere that during the quiescent states may push away the in-flowing matter, letting the NS turn on as a rotationpowered radio pulsar (the radio-ejection mechanism, Kluzniak et al. 1988; Stella et al. 1994; Campana et al. 1998; Burderi et al. 2001; Papitto et al. 2013). Because these accreting MSPs spend most of the time in quiescence, their spin evolution is mainly driven by the magneto-dipole torque, and not by stronger torques associated with disc-field interactions. This implies a slower spin evolution than nuclear MSPs, so that a smaller spin difference might be expected between accreting MSPs and their rotation-powered descendants. The only accreting MSP from which many cycles between outbursts and quiescence have been observed, SAX J1808.4-3658 (see Patruno et al. 2012b, and references therein), spins down at a rate corresponding to a slowdown by just $\sim 5 \mathrm{~Hz}$ over the typical time-scale (100 Myr) associated with the Roche-lobe decoupling phase of more massive donor stars that result in helium white dwarf companions.

Our analysis did not identify a statistically significant difference between the spin distributions of accreting and nuclear MSPs, although the average frequency shown by the latter class is higher by $\sim 150 \mathrm{~Hz}$. However, the differences between these two LMXB classes and the much larger sample of RMSPs can be taken as an indication that a difference between accreting and nuclear MSPs may exist that currently cannot be detected because of the few known objects. A similar conclusion is reached from the significant difference found (95 per cent confidence level) from comparing nuclear MSPs with the whole sample of transitional MSPs and not only accreting MSPs. If nuclear and accreting MSPs are assumed to spin at or close to the equilibrium value 


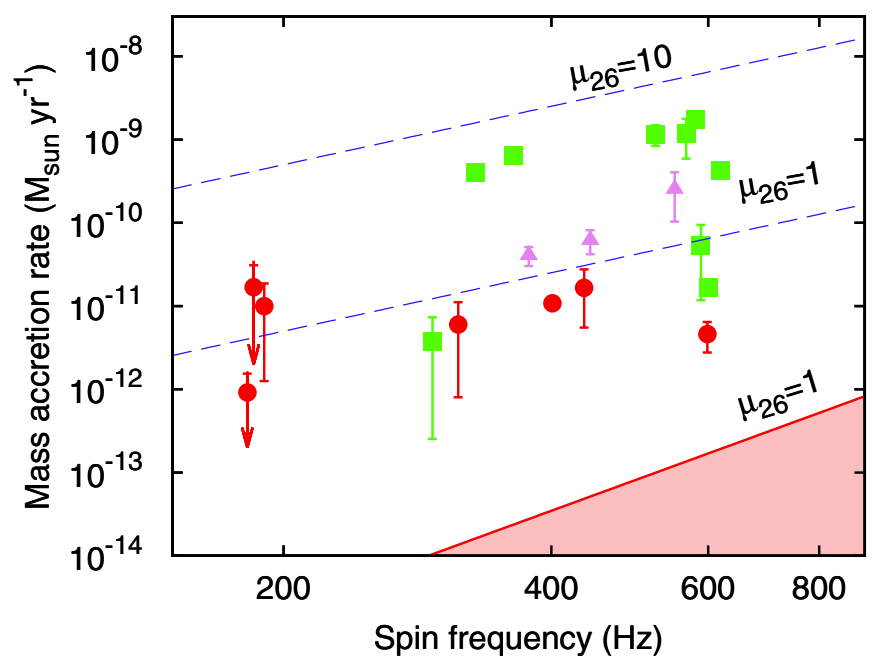

Fig. 4. Long-term mass-accretion rate of millisecond pulsars in the accretion phase, as evaluated by Watts et al. (2008). Accreting-MSPs are plotted as red circles, nuclear MSPs as green squares. Magenta triangles mark the accreting MSPs that showed pulsations only intermittently, and that may have a magnetic field close to the threshold for field burial (Cumming 2008). Blue dashed lines represent the magnetic spin equilibrium lines defined by Eq. (1), evaluated for $\mu_{26}=1$ and $\mu_{26}=10$. The red shaded region defines the parameter space in which a source enters the radio ejector state for $\mu_{26}=1$ (e.g., Burderi et al. 2001).

given by Eq. (1), the contrast between the average frequencies of the two samples can be explained by a difference in the massaccretion rate of $\Delta \dot{M} / \dot{M} \sim 0.8$, and/or in the dipole moment of $\Delta \mu / \mu \sim-0.4$. Nuclear MSPs generally accrete at a higher average rate than accreting MSPs (see green squares and red circles in Fig. 4), and most probably possess a weaker magnetic field because they do not show coherent pulsations. A weaker field might also reflect a higher mass-accretion rate as well because a plasma in-flowing at a high rate diamagnetically screens the magnetic field of the NS below its surface (Bhattacharya \& Srinivasan 1995; Cumming et al. 2001; Cumming 2008). These considerations may be taken as a clue that nuclear MSPs are found in an earlier evolutionary stage that is characterized by a higher average accretion rate (and a consequently lower dipolar magnetic field) and a faster rotation than to accreting MSPs. The latter are instead observed in a phase much closer to the accretion turn-off and to the switch-on of an RMSP, as also demonstrated by the rapid changes of state observed in some of them.

An important role in explaining the different observed spin distributions of some classes might be played by their different evolutionary history. All accreting- and nuclear MSPs for which the orbital period is known belong to systems with $P_{\text {orb }}<1$ day. These binaries originate in systems in which the companion star had a low mass $\left(1-2 M_{\odot}\right)$ and was still on the main sequence, at the onset of mass transfer. The same holds for eclipsing RMSPs, that is, the so-called black widows and redbacks, (cf. Chen et al. 2013, and references therein). In contrast, RMSPs originate in a wider sample of binaries, comprising both systems with low-mass (Tauris \& Savonije 1999) and intermediate-mass (2-6 $M_{\odot}$ ) companions (Tauris et al. 2000). Furthermore, depending on their final $P_{\text {orb }}$ and the composition of the white dwarf companion, in some of these systems the donor star had already evolved off the main sequence at the onset of the mass transfer. Hence, all RMSPs with massive white dwarf companions and RMSPs with helium white dwarf companions in wide orbits $\left(P_{\text {orb }} \gg 1\right.$ day) are only expected to be mildly recycled as a consequence of the relatively short-lived mass-transfer phase in these systems (see Figs. 1 and 9 in Tauris et al. 2012). Given that the observed sample of non-eclipsing RMSPs contains a fraction of such mildly recycled (relatively slow spinning) sources might therefore partly explain why their observed spin distribution is slightly slower than that of eclipsing RMSPs. However, this selection bias probably does not affect the comparison between nuclear and transitional MSPs because all these known sources evolved from low-mass companions in short $\left(P_{\text {orb }}<1\right.$ day) orbital period systems, ensuring a long phase of mass transfer and efficient recycling. Note that an expected short and intense masstransfer phase in wide-orbit systems can explain the hitherto non-detection of accreting- and nuclear MSPs in such binaries as well.

On the other hand, quantifying the impact of instrumental selection biases is more complex and would deserve a population synthesis analysis, which is beyond the scope of this paper. Nevertheless, some conclusions can be drawn on the basis of the observed properties of MSPs in light of the searching strategies actually employed to detect them. Frequency Doppler shifts induced by the orbital motion limit the time interval $T_{\text {best }}$ over which a search for a coherent signal can be performed ( $T_{\text {best }} \propto P_{\text {orb }}^{2 / 3}$; see Eq. (21) in Johnston \& Kulkarni 1991). This significantly reduces the minimum detectable amplitude $A_{\min }$ of accretion powered X-ray pulsations emitted by NS in very-short period binaries $\left(P_{\text {orb }} \lesssim 1 \mathrm{~h}\right)^{4}$, but since the dependence of this effect on the pulsar spin frequency is only weak $\left(A_{\min } \propto v^{1 / 4}\right)$, the resulting bias on the observed spin frequency distribution of accreting MSPs is small. On the other hand, the orbital motion does not affect the detection of nuclear MSPs at all because the burst oscillations are observed at X-ray fluxes $\gtrsim 100$ times higher than accreting MSPs signals, and a detection is achieved in timeintervals of a few seconds (Watts 2012), which is much shorter than the orbital period of the binary. Pulse arrival-time delays induced by orbital motion and by propagation in the ionized interstellar medium also reduce the sensitivity to high-frequency signals in the radio band (Dewey et al. 1985; Hessels et al. 2007). However, recent improvements in data acquisition systems, computational power, and search algorithms have reduced these effects, resulting in doubling the detections of RMSPs in the past five years (Lorimer 2013; Ransom 2013). This increase is also caused by radio follow-up of unidentified $\gamma$-ray Fermi-LAT sources, which now amount to 15 per cent of the total number of RMSPs (Ray et al. 2012; Ransom 2013). As the $\gamma$-ray output of a millisecond pulsar increases with its spin-down power $\left(L_{\gamma} \propto \dot{E}_{\text {sd }}^{a}\right.$, with $a=0.5-1$ and $\dot{E}_{\text {sd }} \propto \mu^{2} v^{4}$; see Abdo et al. 2013), the detection of fast-spinning sources through this method is favoured. During the past five years, the follow-up of Fermi sources determined an eight-fold increase in the number of known Galactic eclipsing RMSP (Roberts 2013), which are significantly faster than the rest of RMSP, as we have shown in this work. Before this overall reduction of the bias against the detection of fast radio pulsars, Lorimer (2013) estimated the peak of the spin frequency distribution of RMSP of the Galactic plane to be $\Delta v \simeq 65 \mathrm{~Hz}$ higher than the observed average. This rapid increase of the MSPs population over the past years then

4 For a $600 \mathrm{~Hz}$ pulsar in a $P_{\text {orb }}=0.5 \mathrm{hr}$ binary observed at an X-ray flux of 200 counts $s^{-1}$ (typical, e.g., of observations performed with EPICpn on-board XMM-Newton), $T_{\text {best }}$ is reduced below $30 \mathrm{~s}$, a time interval that only allows the detection of strong signals with an amplitude larger than $A_{\min } \simeq 12$ per cent (Vaughan et al. 1994), higher than the typical few per cent amplitude signals observed from accreting MSPs (Patruno 2012). A similar result is obtained $\left(A_{\min } \simeq 10\right.$ per cent) if a $300 \mathrm{~Hz}$ pulsar is considered. 
indicates that even if a complete sample of fast pulsars has not been achieved yet, the impact of selection bias has been reduced below this level, $\Delta v$.

In the future, X-ray observatories such as the Large Observatory for X-ray Timing (LOFT Feroci et al. 2012), and to some extent AstroSAT (Agrawal 2006) and NICER (Gendreau et al. 2012), are expected to increase the sensitivity to fast coherent signals from accreting sources up to a factor $\sim 5$. At the same time, further improvement in the computational power and analysis techniques used to discover RMSPs, the numerous candidates identified by Fermi-LAT, and the advent of the Square Kilometre Array (SKA, Smits et al. 2009) will also increase the number of fast radio pulsars in close binary systems. An increase in the number of sources known will be fundamental to constrain the details of pulsar recycling from the point of view of the observed spin distributions.

Acknowledgements. We acknowledge grants AYA2012-39303, SGR2009- 811, and iLINK2011-0303. A.P. is supported by a Juan de la Cierva fellowship. N.R. is supported by a Ramon y Cajal fellowship and by an NWO Vidi Award. D.F.T. is additionally supported by a Friedrich Wilhelm Bessel Award of the Alexander von Humboldt Foundation. T.M.T. gratefully acknowledges financial support and hospitality at both the Argelander-Institut für Astronomie, Universität Bonn and the Max-Planck-Institut für Radioastronomie.

\section{References}

Abdo, A. A., Ajello, M., Allafort, A., et al. 2013, ApJS, 208, 17 Agrawal, P. C. 2006, Adv. Space Res., 38, 2989

Alpar, M. A., Cheng, A. F., Ruderman, M. A., \& Shaham, J. 1982, Nature, 300, 728

Archibald, A. M., Stairs, I. H., Ransom, S. M., et al. 2009, Science, 324, 1411

Bassa, C. G., Patruno, A., Hessels, J. W. T., et al. 2014, MNRAS, submitted [arXiv: 1402.0765]

Bhattacharya, D., \& Srinivasan, G. 1995, in X-ray Binaries, 495

Bhattacharya, D., \& van den Heuvel, E. P. J. 1991, Phys. Rep., 203,

Bogdanov, S., Patruno, A., Archibald, A. M., et al. 2014, ApJ, submitted [arXiv: 1402.6324]

Bozzo, E., Stella, L., Vietri, M., \& Ghosh, P. 2009, A\&A, 493, 809

Breton, R. P., van Kerkwijk, M. H., Roberts, M. S. E., et al. 2013, ApJ, 769, 108

Burderi, L., Possenti, A., D’Antona, F., et al. 2001, ApJ, 560, L71

Burderi, L., Di Salvo, T., D’Antona, F., Robba, N. R., \& Testa, V. 2003, A\&A, 404, L43

Burderi, L., Riggio, A., Di Salvo, T., et al. 2009, A\&A, 496, L17

Burgay, M., Burderi, L., Possenti, A., et al. 2003, ApJ, 589, 902

Campana, S., Colpi, M., Mereghetti, S., Stella, L., \& Tavani, M. 1998, A\&ARv, 8,279

Campana, S., D’Avanzo, P., Casares, J., et al. 2004, ApJ, 614, L49

Chakrabarty, D., Morgan, E. H., Muno, M. P., et al. 2003, Nature, 424, 42

Chen, H.-L., Chen, X., Tauris, T. M., \& Han, Z. 2013, ApJ, 775, 27

Coriat, M., Fender, R. P., \& Dubus, G. 2012, MNRAS, 424, 1991

Cumming, A. 2008, in AIP Conf. Ser. 1068, eds. R. Wijnands, D. Altamirano, P. Soleri, et al., 152

Cumming, A., Zweibel, E., \& Bildsten, L. 2001, ApJ, 557, 958

D’Avanzo, P., Campana, S., Casares, J., et al. 2009, A\&A, 508, 297

Davidson, K., \& Ostriker, J. P. 1973, ApJ, 179, 585

Dewey, R. J., Taylor, J. H., Weisberg, J. M., \& Stokes, G. H. 1985, ApJ, 294, L25

Di Salvo, T., Burderi, L., Riggio, A., Papitto, A., \& Menna, M. T. 2008, MNRAS, 389,1851

Feroci, M., Stella, L., van der Klis, M., et al. 2012, Exp. Astron., 34, 415

Ferrario, L., \& Wickramasinghe, D. 2007, MNRAS, 375, 1009
Ferrigno, C., Bozzo, E., Papitto, A., et al. 2014, A\&A, in press DOI: $10.105 / 0004.6361 / 201322904$

Fruchter, A. S., Stinebring, D. R., \& Taylor, J. H. 1988, Nature, 333, 237

Galloway, D. K., Muno, M. P., Hartman, J. M., Psaltis, D., \& Chakrabarty, D. 2008, ApJS, 179, 360

Gendreau, K. C., Arzoumanian, Z., \& Okajima, T. 2012, in SPIE Conf. Ser., 8443

Ghosh, P. 2007, Rotation and Accretion Powered Pulsars (World Scientific Publishing Co)

Ghosh, P., \& Lamb, F. K. 1979, ApJ, 234, 296

Hartman, J. M., Patruno, A., Chakrabarty, D., et al. 2008, ApJ, 675, 1468

Hartman, J. M., Patruno, A., Chakrabarty, D., et al. 2009, ApJ, 702, 1673

Hartman, J. M., Galloway, D. K., \& Chakrabarty, D. 2011, ApJ, 726, 26

Hessels, J. W. T. 2008, in AIP Conf. Ser. 1068, eds. R. Wijnands, D. Altamirano, P. Soleri, et al., 130

Hessels, J. W. T., Ransom, S. M., Stairs, I. H., Kaspi, V. M., \& Freire, P. C. C. 2007, ApJ, 670, 363

Iacolina, M. N., Burgay, M., Burderi, L., Possenti, A., \& di Salvo, T. 2009, A\&A, 497, 445

Iacolina, M. N., Burgay, M., Burderi, L., Possenti, A., \& di Salvo, T. 2010, A\&A, 519, A13

Illarionov, A. F., \& Sunyaev, R. A. 1975, A\&A, 39, 185

Johnston, H. M., \& Kulkarni, S. R. 1991, ApJ, 368, 504

Kluźniak, W., \& Rappaport, S. 2007, ApJ, 671, 1990

Kluzniak, W., Ruderman, M., Shaham, J., \& Tavani, M. 1988, Nature, 334, 225

Kuiper, N. H. 1960, Proc. Kon. Ned. Akad. Wetensch., A, 63

Linares, M., Bahramian, A., Heinke, C., et al. 2014, MNRAS, 438, 251

Lorimer, D. R. 2013, in IAU Symp., 291, 237

Pallanca, C., Dalessandro, E., Ferraro, F. R., Lanzoni, B., \& Beccari, G. 2013, ApJ, 773, 122

Paltani, S. 2004, A\&A, 420, 789

Papitto, A., Riggio, A., Burderi, L., et al. 2011, A\&A, 528, A55

Papitto, A., Ferrigno, C., Bozzo, E., et al. 2013, Nature, 501, 517

Papitto, A., Torres, D. F., \& Li, J. 2014, MNRAS, 438, 2105

Patruno, A. 2010, ApJ, 722, 909

Patruno, A. 2012, ApJ, 753, L12

Patruno, A., \& Watts, A. L. 2012 [arXiv: 1206.2727]

Patruno, A., Alpar, M. A., van der Klis, M., \& van den Heuvel, E. P. J. 2012a, ApJ, 752, 33

Patruno, A., Bult, P., Gopakumar, A., et al. 2012b, ApJ, 746, L27

Patruno, A., Archibald, A. M., Hessels, J. W. T., et al. 2014, ApJ, 781, L3

Radhakrishnan, V., \& Srinivasan, G. 1982, Current Sci., 51, 1096

Ransom, S. M. 2013, in IAU Symp., 291, 3

Rappaport, S. A., Fregeau, J. M., \& Spruit, H. 2004, ApJ, 606, 436

Ray, P. S., Abdo, A. A., Parent, D., et al. 2012 [arXiv: 1205. 3089]

Riggio, A., Burderi, L., Di Salvo, T., et al. 2011, A\&A, 531, A140

Roberts, M. S. E. 2013, in IAU Symp., 291, 127

Roy, J., Bhattacharyya, B., \& Ray, P. S. 2014, ATel, 5890, 1

Ruderman, M., Shaham, J., \& Tavani, M. 1989, ApJ, 336, 507

Smits, R., Kramer, M., Stappers, B., et al. 2009, A\&A, 493, 1161

Stappers, B. W., Archibald, A. M., Hessels, J. W. T., et al. 2013, ApJ, submitted [arXiv: 1311.7506]

Stella, L., Campana, S., Colpi, M., Mereghetti, S., \& Tavani, M. 1994, ApJ, 423, L47

Stephens, M. A. 1974, J. Am. Statist. Assoc., 69, 730

Tauris, T. M. 2012, Science, 335, 561

Tauris, T. M., \& Savonije, G. J. 1999, A\&A, 350, 928

Tauris, T. M., van den Heuvel, E. P. J., \& Savonije, G. J. 2000, ApJ, 530, L93

Tauris, T. M., Langer, N., \& Kramer, M. 2012, MNRAS, 425, 1601

Vaughan, B. A., van der Klis, M., Wood, K. S., et al. 1994, ApJ, 435, 362

Verbunt, F., van den Heuvel, E. P. J., van Paradijs, J., \& Rappaport, S. A. 1987, Nature, 329, 312

Wang, Y.-M. 1987, A\&A, 183, 257

Watts, A. L. 2012, ARA\&A, 50, 609

Watts, A. L., Krishnan, B., Bildsten, L., \& Schutz, B. F. 2008, MNRAS, 389, 839

Watts, A. L., Altamirano, D., Linares, M., et al. 2009, ApJ, 698, L174

Wijnands, R., \& van der Klis, M. 1998, Nature, 394, 344

Xing, Y., \& Wang, Z. 2013, ApJ, 769, 119 